\title{
Destabilizing factors in operation of short-cavity He-Ne laser
}

\author{
G.L. Kononchuk, V.V. Stukalenko \\ Physics Department, Kyiv National Taras Shevchenko University, 6 Akademician Glushkov prospect, 03127 Kyiv, Ukraine \\ E-mail:stvik@ukr.net
}

\begin{abstract}
The influence of longitudinal manetic field (MF) on a position of polarization planes (PPs) of the main modes $(\lambda=0.63 \mu \mathrm{m})$ in dependency on the generation frequency and off-axis mode presence was investigated in this work. It was shown that mode PPs are strictly orthogonal only in their symmetrical position in the amplification contour. In other spectral ranges PP position depends on the value of cavity anisotropy and local MF. Local MF action is equivalent to that of homogeneous MF. Characteristics of axis modes (intensity, PP position, optical frequency and especially an inter-mode beating frequency) are destabilized by modes of higher orders, dynamics of which possesses a random character. Nonlinear interaction between modes and a strong dependency of their characteristics on a local MF make stabilization system reliable operation impossible, if these systems are based only on internal properties of laser emission. The high sensitivity of the polarization state to the local MF can be used for broad-band polarizational modulation of laser emission.
\end{abstract}

Keywords: gas laser, stabilization, local magnetic field, polarization plane, mode nonorthogonality, intermode beating.

Paper received 09.06.03; revised manuscript received 13.06.03; accepted for publication 16.06.03.

\section{Introduction}

Investigation of magnetic field action on gas-discharge laser emission has a long history, however, the interest to this topic does not wane due to the well-known diversity of features accompaning this action. In practice, a most promising are desk-size two-mode lasers of LGN-208 type with the short quasi-isotropic cavity $(L=0.24 \mathrm{~m})$, which simultaneously generate two orthogonally polarized modes with the intermode separation $\Delta v=c / 2 L=$ $=640 \mathrm{MHz}$. Rather often polarization and frequency properties of these lasers are tried to be used for stabilization of output laser parameters, namely: the emission frequency and power, but, as was shown in [1-4], there was no reliable substantiation for such application.

Our experimental investigations were performed for ascertaining possibilities to use laser intrinsic characteristics for stabilization of emission output parameters. Our special attention was paid to phenomena that accompany the cavity thermal extension (instability of a mode composition) and the influence of weak local magnetic field (MF) on emission polarization characteristics. The latter is important, because the Earth magnetic field essentially impacts the operation of the laser with a quasiisotropic cavity. In particular, some parts of the facility possessing ferromagnetic properties can bring all the efforts for laser parameter stabilization to naught.

\section{Experimental results}

Our experimental facility enables to determine position of the polarization plane (PP) for every mode, it is a combination of a polarimeter and two spectrometers, which is capable to synchronically register mode optical spectra in orthogonal polarizations and intermode beating frequency (IBF) spectra in the microwave range during the laser thermal extension [5]. Initial experimental data were introduced into computer and treated using specially developed software. Fig. 1 shows two fragments of the synchronical records of the generation spectrum change registered in two standard positions conventionally named as the vertical $(\mathrm{V})$ and horizontal $(\mathrm{H})$.

When modes cross the amplification contour in the course of cavity thermal extension at the initial stage (positions V1, H2), planes of the electric vector oscillations in both modes coincide with directions of oscillations that are registered by respective detectors (two photomultipliers with two-beam Glan-Thomson prism). The spectral range of active medium amplification is laid 


\section{G.L. Kononchuk, V.V. Stukalenko: Destabilizing factors in operation of short-cavity ...}

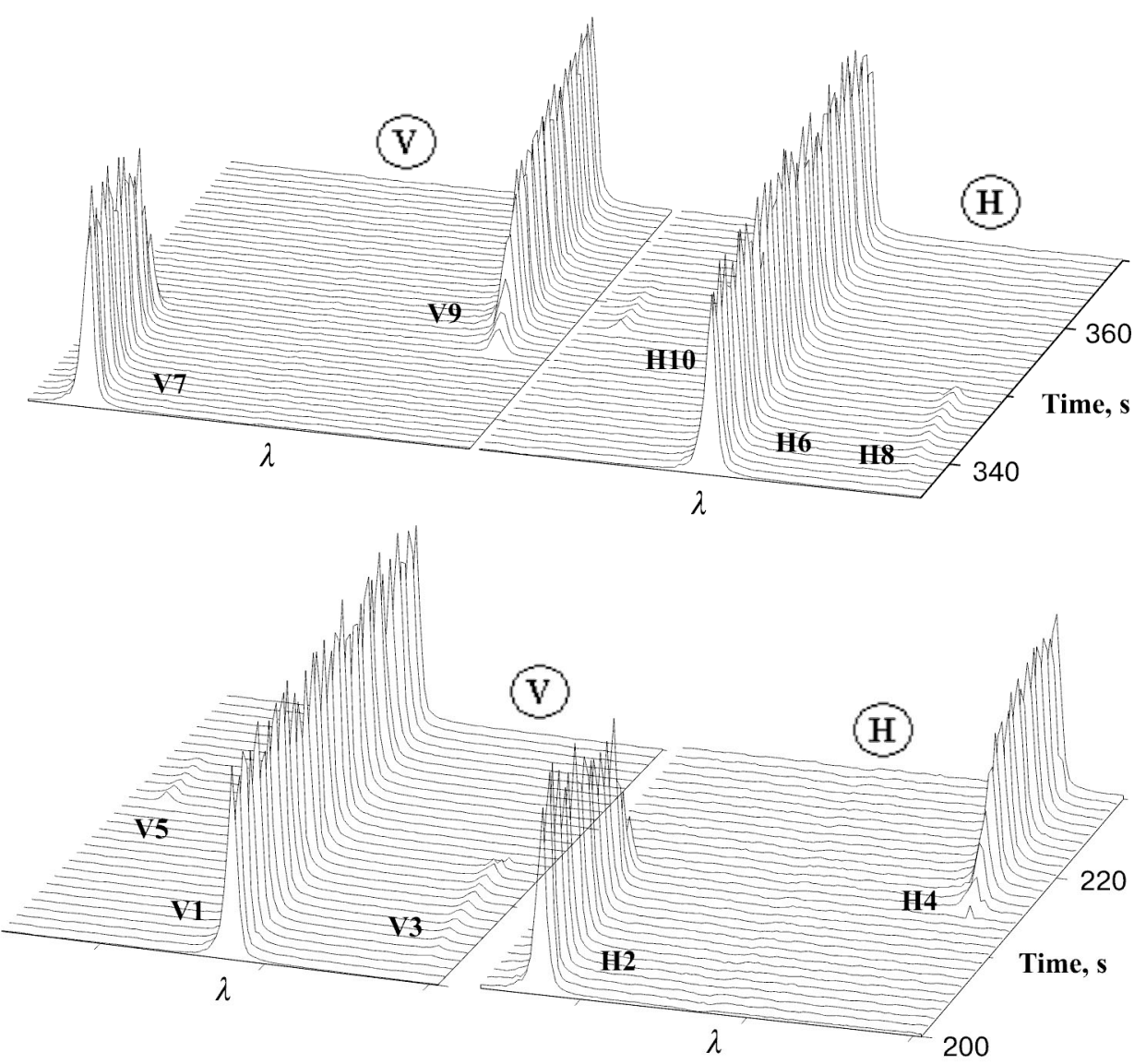

Fig. 1. Two fragments of one continuous record of orthogonal mode intensity development when the cavity is extended. V1 mode is at the centre of the amplification contour, while $\mathrm{H} 2$ mode is at the low-frequency slope of it. The decay of $\mathrm{H} 2$ mode is accompanied with a short-term appearance of V3 mode at the high-frequency slope, which changes its polarization very soon (H4). At the same time, the mode of $\mathrm{H} 2$ frequency transfers into V-polarization and disappears in it (V5). Within a period close to $100 \mathrm{~s}$, V1 mode shifts into V7 state, H4 mode - into H6 state, the character of mode frequency and polarization changes is fully repreduced (V7 $\rightarrow$ H10; $\mathrm{H} 8 \rightarrow \mathrm{V} 9)$.

off along the $\lambda$-axis, another horizontal axis represents the registration time (number of scanning), while the vertical axis shows the light intensity. At $t=200 \mathrm{~s}$, each mode exists only in one polarization, but the position of its PP is changed by the orthogonal one when the mode is found near the edge of the contour.

It is reasonable to state that the mode has the tendency to arise and vanish in the same polarization as the central mode, which is in the range of the Lamb dip at this instant. This effect can be observed in both polarizations, and none of polarizations has advantages. At the same time, the cavity quality factor depends on the polarization, that is why a minor distinction in complex refraction indexes causes non-equidistant intermode intervals and jumps in IBF spectrum $[5,6]$.

Synchronous registration of the emission spectra $I_{1}(\lambda)$, $I_{2}(\lambda)$ in two orthogonal planes enables to follow PP positions for each mode in detail. The angle of PP turn $\varphi(\lambda)$ can be calculated using the formula $\varphi(\lambda)=\operatorname{arctg}\left(I_{2} / I_{1}\right)^{1 / 2}$. Our experiments were carried out both by using magnetic insulation of the laser tube from external magnetic fields (with a multilayer screen of permalloy) and applying a local MF to the laser.

It was observed that projections of the light wave electrical vector onto two basic directions are changed in dependence on the generation frequency even in zero field, i.e., the position of mode PP is not kept constant. The maximum PP "swinging" takes place at the centre of the amplification contour, and has common value $\pm 2^{\circ}$, although it can be larger. It is in this spectral position (the Lamb dip) the change of the mode polarization can take place, however, this jump can fail.

In the course of the cavity extension, modes are shifted along the amplification contour from the high-frequency slope to the low-frequency one. The mode PP behavior depends on what mode ( $\mathrm{V}$ or $\mathrm{H})$ occupies the low-frequency slope at the moment ("leading mode"). From the physical viewpoint, these two cases differ by the value of the intermode interval: in the first case, it is $100-200 \mathrm{kHz}$ shorter than in the second one. Fig. 2 illustrates the character of MF action on the mode PP. The leading mode in the cases $a$ and $b$ is the mode of $\mathrm{H}$-type, and the driven 


\section{G.L. Kononchuk, V.V. Stukalenko: Destabilizing factors in operation of short-cavity ...}

mode is of V-type. The polarization jump takes place at the center of the spectral line - the driven mode became leading, then the situation repeats, $a$ and $b$ cases are distinguished by MF direction. In Fig. $2 c, \mathrm{~V}$-mode is leading. In Fig. $2 d$, the polarization jump did not take place: first, H-mode is leading, and once it dissappears V-mode becomes leading. As a consequence, one can observe a combination of two previous cases. $\varphi(v)$-functions are different in all cases.

The maximum PP turn takes place, when the mode is in the center of the line. In this case, the peripheral mode is turned by approximately $\pi / 2$ relatively to PP of the central mode, that is, with applying MF, these modes have the same direction of PP turn. With symmetrical localization of the modes relatively to the line centre (at the points $\pm 320 \mathrm{MHz}$ ), the PP turn is absent, and polarization planes of both modes coincide with the basic ones (without MF) in all cases.

The net effect on a mode polarization for local magnetic fields acting along the gas discharge has the additive character. We ascertained it in the following manner. The local magnetic field was created using the coil with the diameter $58 \mathrm{~mm}$ and width $17 \mathrm{~mm}$. The coil was placed at various sections along the laser tube (discharge) with separations of $3 \mathrm{~cm}$, sampling being made from the tube end (from the side of back mirror). In every position of the coil, first, we registered the behavior of the mode PP without local MF, and then - with it. The difference between two records stemmed from the magnetic field impact.
It is obvious that in every case MF distribution along the discharge channel is different, however, the effective field $H_{\text {eff }}$ (calculated per one run of the beam in the cavity) can be determined using a convolution of two functions - MF distribution $H(x)$ and the amplification factor along the gas discharge of this configuration. This effective field should depend on the mutual disposition of the coil and laser. These dependences on the local MF position(see Fig. 3) are calculated and measured for the laser LGN-208B and the coil. Calculated results are depicted with the solid line, while the experimental ones with dots. These illustrate values of PP turn angles for both orthogonal modes being in the centre of the amplification contour. The dependences are normalized to unity.

As seen from the figure, the calculated effective local field correlates well with the value of PP turn angle for the modes of both polarizations. It is indicative of the validity to search $H_{\text {eff }}$ as the weighted sum, i.e., of the additive MF action on the mode PP turn. Obviously, the discharge central part is the most sensitive to the local MF. This circumstance efficiently simplifies the task of a laser emission wide-band modulation, as it enables us to considerably expand the modulation frequency range into the high-frequency side.

We did not find any qualitative difference in the mode behavior when applying homogeneous or local MF. Approximately symmetric behavior of curves 3 and 4 in Fig. 2 confirms orthogonality of mode's PP, but detail calculations show that it is wrong. Synchronous information about the mode PP angle in each spectral posi-
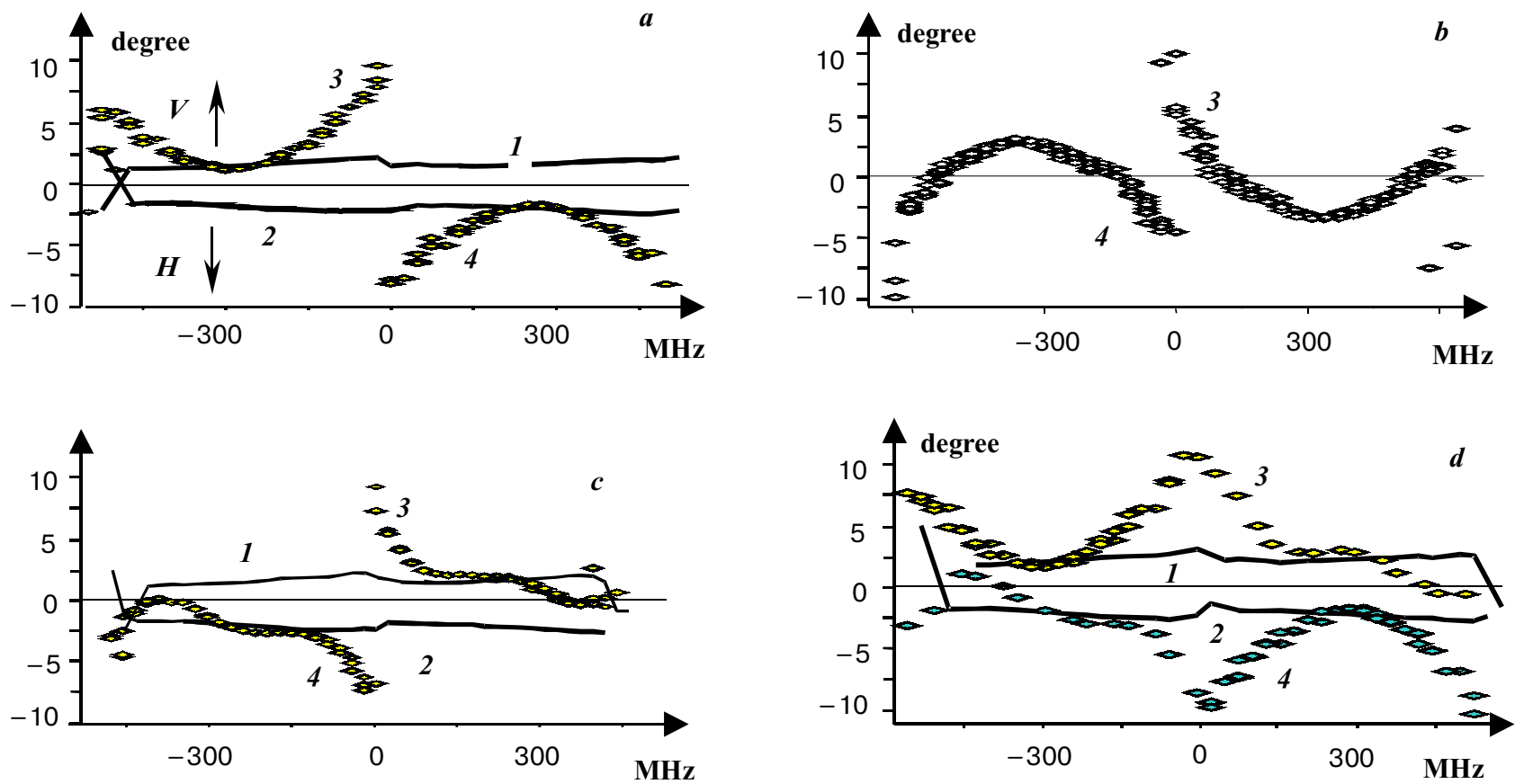

Fig. 2. Spectral dependences of the mode PP turn without magnetic field $(1,2)$ and with the applied one (3, 4). Arrows show the direction of the respective mode ( $\mathrm{V}$ or $\mathrm{H}) \mathrm{PP}$ turn to the right (following to the beam). All the dependences are artificially shifted to the right using the detector turn by approximately $2^{\circ}$. Shifting the mode frequency was made from the right to the left. 


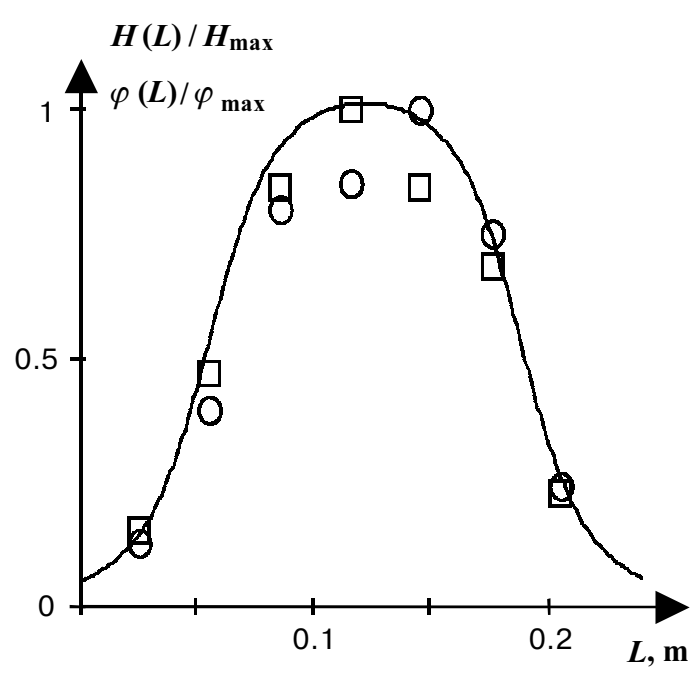

Fig. 3. PP turn under the action of longitudinal magnetic field in the dependence of the coil localization.

tion allows to exactly determine the angle between PPs of the quasi-orthogonal modes and find its distinction of $\pi / 2$. Fig. $4 a$ illustrates this dependence in two cases when the leading mode is $\mathrm{V}(1)$ and $\mathrm{H}(2)$, while Fig. $4 b$ shows that the angle between PPs of the quasi-orthogonal modes is not equal to $\pi / 2$.

The nonmonotonic behavior of these dependences in the range $0 \ldots 640 \mathrm{MHz}$ is caused by instability of PP position for any mode when it is in the Lamb dip. One can draw the conclusion that slight closing in these modes in the frequency range (by $\sim 0.2 \mathrm{MHz}$ ) results in better orthogonality than their separation. However, this fact needs serious theoretical substantiation. It practically means that even MF variations bound to the laser orien-
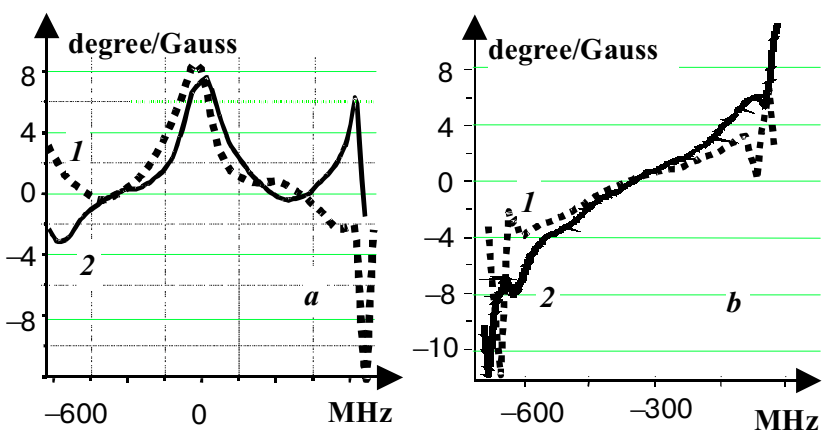

Fig. 4. Spectral dependences of the mode PP turn in magnetic field $(a)$, and the mode angle of non-orthogonality $(b)$. The leading mode is $\mathrm{V}$ mode (I) or $\mathrm{H}$ one (2).

tation relatively to the Earth magnetic field or laboratory equipment can significantly destroy interrelation between quasi-orthogonal mode PPs.

It is absolutely clear that sensitivity of the mode PP position to an external field is determined by the distinction of cavity losses in orthogonal polarizations: the smaller is the distinction, the higher is the sensitivity. In any case, in a monotypic cavity with one Brewster window (LGN-207B), based on our data, this sensitivity is, at least, one thousand times lower. It means that the dependence of Fig. 4 type can look differently for another laser.

As mentioned in [5], one more destabilizing factor is the instability of a mode composition. Frequently in the emission of a single-mode (in accord with its certificate) laser, one can observe modes of higher orders. Fig. 5 shows the fragment of the generation spectrum recorded for $110 \mathrm{~s}$ at the moment of reaching the stationary regime. Appearance of each new generation frequency

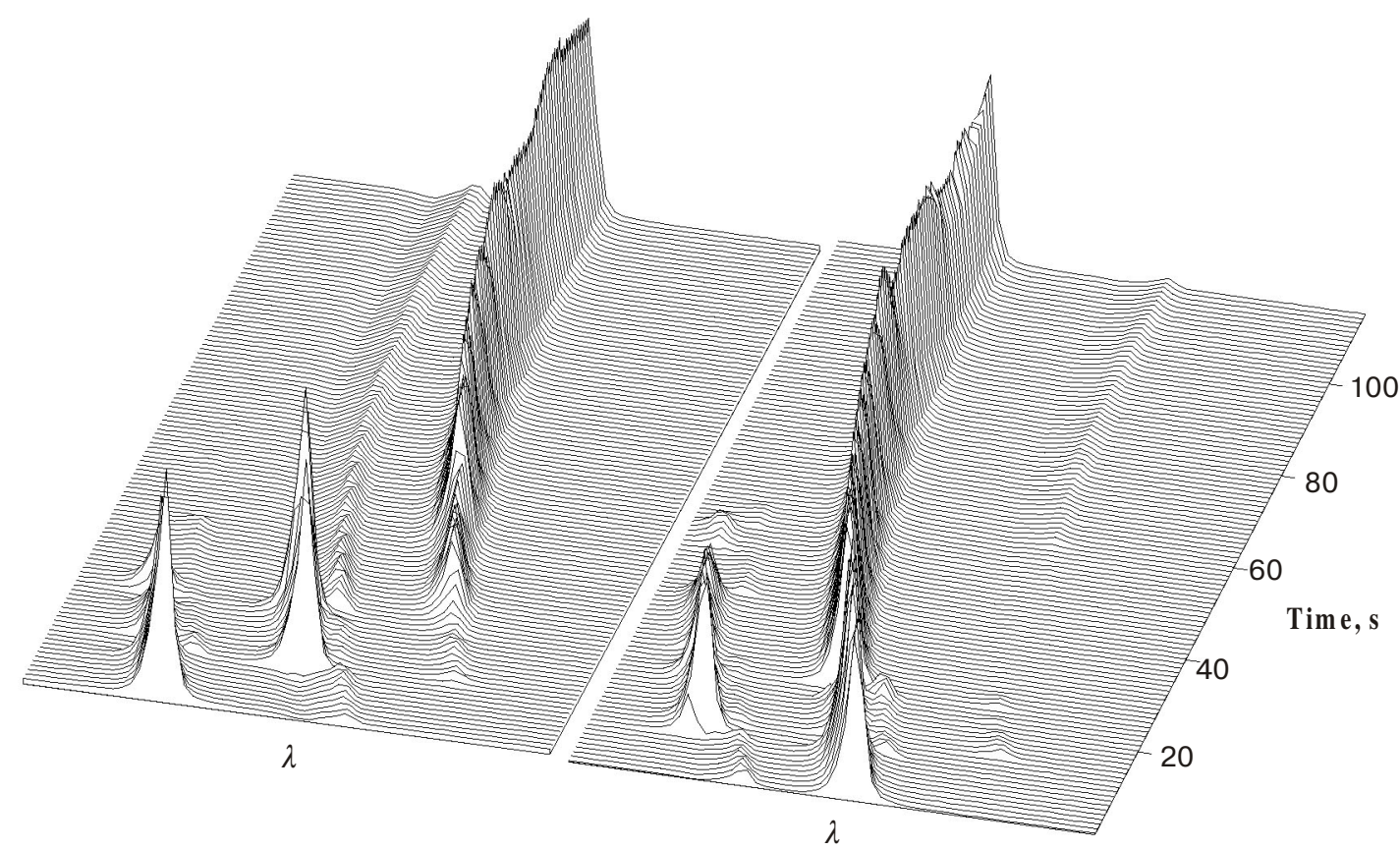

Fig. 5. Spectrum of generation in two mutually orthogonal polarizations at the presence of off-axis modes. The off-axis mode intensity is one order less than that of the main ones, changes of their frequency and polarization being of a chaotical character. 

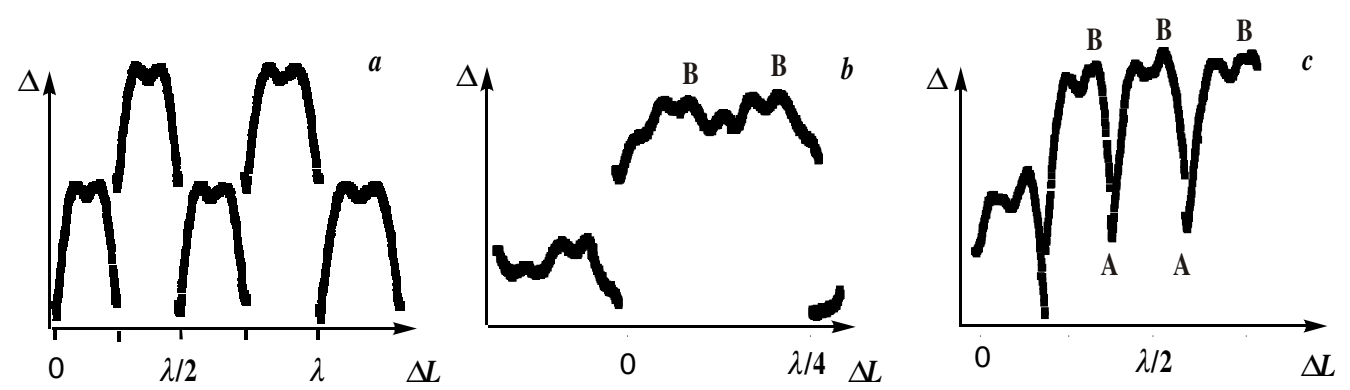

Fig. 6. Inter-mode beating frequency changes as a function of the cavity extension at mode polarization jumps (A) at the centre of the amplification contour as well as at presence of off-axis modes (B).

causes a deformation in Bennet's dips that dynamically influence the course of the refraction index dispersion within amplification contour. This impact sharply destabilizes all other modes by their frequency, power and polarization. This re-distribution of the energy between modes is accompanied with frequency jumps that can be observed in the most simple way through the spectrum of inter-mode beating.

Several typical cases are shown in Fig. 6: $a$-corresponds to regular generation of two quasi- orthogonal modes, each of them exists in its proper polarization for all frequencies of the amplification contour; $b$-axis modes (similar to $a$ ) do not change their polarization, but the modes of higher orders arise chaotically; $c$ - the jump into the other polarization takes place, when the mode is in the Lamb dip (A); besides, the off-axis mode arises (B). Practically, in no cases there is one-to-one correspondence between the generation frequency and IBF one. It means that IBF cannot serve as an input signal of the optical mode frequency stabilization system.

\section{Conclusions}

Because of non-ideal cavity isotropy, there is a own net of practically equidistant frequencies in every polarization, and these nets being shifted relative to each other. As a result, this two-mode generation in the quasi-isotropic cavity can be one of two types, with the inter-mode separation between quasi-orthogonal modes, which is smaller or larger than the separation in each net. When the real quasi-isotropic cavity is thermally extended, its modes are characterized by more sophisticated frequency and polarization dynamics of its development. In particular, every mode possesses a tendency to appear (and disappear) at the edge of the amplification contour just in the polarization that is already inherent to the central mode.

Position of polarization plane for each mode are greatly sensitive to an external magnetic field and can be changed by several degrees at the induction close to 1 Oersted, this sensitivity being spectrally dependent. In a general case, the modes are not orthogonal, the angle between PP depends on the mode frequency and magnetic field value. Only at the symmetrical mode position within the amplification contour, the modes are strictly orthogonal, and PP position does not depend on an external MF. For both above mentioned modes, the spectral behavior of PP position in the external MF is also distinguished.

Modes of higher orders, which arise in non-controlled manner, possessing a random polarization and power, can influence all the characteristics of axis modes and change their intensity, PP positions and IBF. This influence can take place due to additional Bennet's dips and related with them dispersion of the active medium refractive index.

Thus, polarizational, amplitude and mode instabilities of He-Ne laser emission result in failures of any stabilization system based on internal cavity properties. Some of these systems, which are made and tested using separate laser samples, can attract attention due to their simplicity, cheapness, but wide implementation will most likely be of little use. It is noteworthy that even being made in several samples, all these systems, despite specific way of designing, should include units of thermal stabilization and discharge current control, otherwise variations in the Doppler contour characteristics will cause fluctuations of the abnormal dispersion [7], which results in the amplitude-frequency instability.

\section{References}

1. A.N. Vlasov, V.A. Perebyakin, S.Yu. Polyakov, V.E. Privalov // Kvantovaya elektronika, 13(2), pp. 320-325 (1986), (in Russian).

2. C. Ciddor, K.M. Duffy // J. Phys. E: Sci. instrum. 16(12), pp. 1223-1227 (1983).

3. A.N. Vlasov, V.E. Privalov // Pribory i systemy upravleniya, №10, pp. 40-42 (1999), (in Russian).

4. A. Sasaki, T. Okada, J. Kawai, H. Aoyama // Appl. Phys. Lett., 61(10), pp. 1151-1153 (1992).

5. G.L. Kononchuk, V.V. Stukalenko // Ukrainsky fizychnyi zhurnal, 48(5), pp. 417-423 (2003), (in Ukrainian).

6. G.L. Kononchuk // Zhurnal fizychnykh doslidzhen', 3(4), pp. 451-455 (1999), (in Ukrainian).

7. G.L. Kononchuk, V.V. Stukalenko, Yu.G. Terentyeva. Influence of the active medium temperature on gas laser intermode intervals // Naukovi zapysky. Ser. fiz.-mat. nauky. Ed. by National University Kyiv-Mogyla Academy, №1, p. 83-90 (2002), (in Ukrainian). 